Pensamiento Crítico Vol. 20 N 1, pp. 43-63

\title{
El proceso analítico, considerando lo aritmomórfico, la aducción y la complejidad. La importancia de la investigación teórica en la ciencia económica: Reflexiones sobre la contribución de Georgescu-Roegen*
}

The analytical process, considering aritmomorphic, adduction and complexity. The significance of the theoretical research on economic science:

Reflections on Georgescu-Roegen's contribution

[Recepción: Marzo de 2015/ Conformidad: Mayo 2015]

Juan Manuel Eliud Cisneros García**

* Expreso mis agradecimientos a Manuel Cisneros, mi padre, por el intercambio de ideas en el tema; también a los colegas Eloy Ávalos y Hugo Sánchez por los aportes brindados en diversas conversaciones. Ante la probable presencia de errores en este documento debo exculpar a los mencionados, cualquier falta es de mi exclusiva responsabilidad.

** Profesor Auxiliar del Departamento de Economía de la UNMSM. B. Sc. Economía, UNMSM. Maestría en Economía (c), Universidad Nacional de La Plata. Maestría en Economía con mención en Desarrollo Empresarial y Regional (c), UNMSM. Maestría en Sociología con mención en Educación y Desarrollo (c), UNMSM. Investigador asociado al Instituto de Investigaciones Económicas (IEE). Investigador asociado al Instituto de Estudios Sociales del Rímac (IESR). Correo electrónico: jcisnerosg@unmsm.edu.pe 


\title{
Pensamiento Crítico Vol. 20. NoI
}

\section{RESUMEN}

La idea de proceso analítico resulta imprescindible para poder entender el quehacer de la investigación teórica en la Ciencia Económica, pues sólo son explicables aquellos temas que pueden ser manifestados como un proceso. La explicación de un conjunto de hechos sociales relacionados con alguna actividad económica sólo puede realizarse con una teoría, la cual se plantea considerando un proceso analítico. La complejidad de una realidad social debe ser simplificada por abstracción, para ello se requiere tener presente dos nociones fundamentales: lo aritmomórfico y la aducción. Lo planteado en el presente escrito está basado en la propuesta epistemológica desarrollada por Georgescu-Roegen.

Palabras Claves: Proceso, aducción, aritmomórfico, complejidad, ciencia económica, investigación.

Clasificación JEL: B40, B41.

\begin{abstract}
The notion of analytical process is essential to understand the task of theoretical research in Economic Science, because it is explicable only those items that can be expressed as a process. The explanation for a set of social facts related to any economic activity only can be performed with a theory, which arises considering an analytical process. The complexity of social reality must be simplified by abstraction, this requires to recognize two fundamental notions: the aritmomorphic and the adduction. The issues raised in this paper are based on the epistemological proposal developed by Georgescu-Roegen.
\end{abstract}

Keywords: Process, adduction, complexity, aritmomorphic, economic science, research. 


\section{Juan Manuel Eliud Cisneros García}

\section{Introducción}

La idea de proceso analítico es un tema al que se ha brindado poca atención, Georgescu-Roegen menciona que si bien este término se difundió con una gran rapidez en la literatura científica, ello ocurrió sin que haya sido definido de modo preciso. Nuestra intención es contribuir a la precisión del referido término en el campo de la Ciencia Económica y mostrar la importancia que tiene en la investigación teórica. Por ello, se presenta un conjunto de conceptos que consideramos importantes: lo aritmomórfico (término planteado por Georgescu-Roegen), el cual se contrapone a la idea de dialéctica (vinculada con la noción de proceso), pero permite realizar la separación de manera discreta de al menos dos conceptos;la aducción, un término ciertamente poco difundido, pero que permitirá dejar de involucrar una investigación con el inductivismo (y los problemas que éste tiene), así como permitir vincular la Ciencia Económica con otras ciencias; ambos son importantes para fines de la abstracción. La noción de complejidad es relevante en el presente escrito.

Entendemos que la ciencia está basada en el estudio de procesos analíticos, los cuales tienen una imagen compuesta por: el límite analítico (frontera y duración del proceso) y el suceso; se profundiza en el tema referido a las coordenadas analíticas de un proceso,los elementos exógenos y endógenos; y la flecha del tiempo que permite establecer la irreversibilidad.

Considerando lo mencionado y recalcando la necesidad de la existencia de regularidades empíricas, se indican los criterios para identificar que hechos son investigables y cuales no lo son en el campo de la Ciencia Económica.

En la parte final del escrito se incorpora la noción de teoría, la cual permitirá explicar el resultado de un proceso. Entendemos que en la investigación en la Ciencia Económica, actualmente, existen dos tendencias: la investigación teórica y la investigación empírica.

\section{Aritmomófico}

La noción de aritmomófico hace referencia a la separación de manera abstracta ${ }^{1}$

1 La abstracción entendida como la separación mental de los elementos que son considerados por el investigador como los más importantes respecto a una realidad. Es importante recalcar que ello implica una acción arbitraria, por ello debe considerarse la existencia de un dualismo epistemológico (Encinas, 1992: 24); además se puede considerar cierto nivel de intencionalidad referido a intereses no académicos 


\section{Pensamiento Crítico Vol. 20. NoI}

de al menos dos elementos, permite considerarlos aisladamente uno del otro, de manera discreta, no continua, individualizando cada elemento sin que uno esté superpuesto al otro, ello resulta básico para poder establecer orden lógico entre proposiciones. «Dado que todo número real específico constituye el ejemplo más elemental de concepto discretamente diferenciado, propongo que se denomine a tal concepto aritmomórfico. En efecto, a pesar de emplear el término «continuo» para el conjunto de todos los números reales, dentro del continuo cada número real conserva una individualidad diferenciada idéntica en todos los aspectos a la de un número entero dentro de una secuencia de números naturales. (...). En la Lógica, «es» y «no es», «corresponde» y «no corresponde», «algunos» $\mathrm{y}$ «todos» son también discretamente diferenciados. (...) Para decirlo más directamente, los conceptos aritmomórficos no se superponen. Es precisamente esta peculiar (y restrictiva) propiedad de los materiales con los que puede trabajar la Lógica lo que explica su tremenda eficacia: sin esta propiedad, no podríamos efectuar cálculos, ni hacer silogismos, ni construir una ciencia teórica. Ahora bien, como sucede con todos los poderes, el de la Lógica se encuentra igualmente limitado por su propio fundamento.» (Georgescu-Roegen, 1996: 93)

Existe una notable distinción entre el concepto aritmomórfico ${ }^{2}$ y el concepto dialéctico ${ }^{3}$, según Georgescu-Roegen el carácter aritmomórfico de un concepto se relaciona con el hecho de que hay una frontera final, discreta, entre lo que es y lo que no lo es. La capacidad para establecer una relación entre un conjunto de conceptos y una escala numérica permite establecer el carácter aritmomórfico de un concepto. Resalta el hecho de que los conceptos dialécticos sean de orden cualitativo, además que los conceptos cuantitativos se construyen a partir de los conceptos cualitativos, estos últimos aparecen de manera frecuente en diversas teorías que son parte de la Ciencia Económica y complican la aplicación del razonamiento aritmomórfico. La importancia de los modelos aritmomórficos radica en que permiten testear los razonamientos dialécticos ${ }^{4}$.

(Gonzáles, 2004: 23-25).

2 La palabra aritmomórfico no se encuentra en el Diccionario de la Real Academia Española (DRAE). En el idioma inglés, la palabra aritmomorphic tampoco se encuentra en diccionarios tales como: Cambridge, Collins u Oxford. En francés, arithmomorphique tampoco está presente entre las palabras de los diccionarios más reconocidos de dicho idioma.

3 «In GR's terminology, models, based on logic, are aritmomorphic: discrete, sharp representations of the reality which leave qualitative attributes outside the description; the reality is a dialectical continuum, i.e., it is a continuum of entities that are different yet non-completely distinguishable one from the other (Fontini, 2005: 5)»

4 Véase: Dulbecco y Garrouste (2004: 11-12) y Cisneros (2014b). 


\section{Juan Manuel Eliud Cisneros García}

En la ciencia económica el concepto aritmomórfico permite la separación, por abstracción, de los hechos de la realidad que se buscan conocer, permite identificar elementos que serán investigados, plantear proposiciones fundamentales; así se puede establecer orden lógico, por tanto existirán teorías, ello hará posible el planteamiento de modelos económicos (teorías económicas operativizadas por la incorporación de supuestos auxiliares $)^{5}$, al existir variables, se podrán plantear relaciones de causalidad entre ellas, las mismas que tendrán que ser contrastadas con la base de datos correspondiente.

\section{Aducción}

Para realizar investigación teórica en la Ciencia Económica se requiere hacer uso de la abstracción, la pregunta que surge es ćqué existe antes de ello?, definitivamente el hecho de empezar a separar mentalmente los hechos de la realidad asignándoles niveles de importancia diferenciado no es un acto espontáneo. Algunos podrían asumir que la acumulación de observaciones y el planteamiento de proposiciones particulares y la posterior generalización de éstas es el inicio, es decir, la inducción como paso previo, pero ello no es lógicamente correcto debido a los problemas que muestra, por ello no es aceptable como argumento lógico demostrativo (Popper, 2002). Se puede considerar que las ideas previas provienen de un argumento no lógico, se puede considerar la experiencia, la intuición, las ideas provenientes de otras disciplinas filosóficas y científicas, incluso el conocimiento de un tema basado en ideas no vinculadas con el mundo occidental, entre otros recursos con los cuales cuente un investigador 6 . "Grandes confusiones se evitarían, sólo con que consiguiésemos garantizar la utilización lingüística del término "aducción" para las formas de razonamiento no-demostrativas, y a las que vulgarmente se aplica el término "inducción" podríamos evitar una gran cantidad de malentendidos.» ${ }^{7}$ (Blaug, 1997: 17) 8 [La traducción es nuestra]

5 Lo cual permitirá que en la ciencia económica se haga uso de tópicos de la matemática, nótese que éstos constituyen un medio de ayuda para seguir avanzando en la investigación teórica.

6 Como ejemplo de aducción, resulte interesante revisar el procedimiento que propone Varian (1997) respecto a cómo plantear un modelo económico, no obstante el autor en mención no hace referencia textual alguna al término aducción.

7 Blaug indica (op. cit.: 17) que el término aparece en: Black (1970: 137).

8 Blaug recalca que debe considerarse a la aducción como: «(...) la operación no lógica que permite saltar desde el caos que es el mundo real a la corazonada [o sospecha, hunch] que supone una conjetura tentativa respecto de la relación que realmente existe entre un conjunto de variables relevantes. La cuestión de cómo se produce dicho salto pertenece al contexto de la lógica del descubrimiento, y puede que no sea conveniente dejar de lado despectivamente este tipo de contexto, como los positivistas, e 


\section{Pensamiento Crítico Vol. 20. NI}

Entendemos que la aducción permitirá un mayor acercamiento con las otras ciencias sociales, ello resultará importante en un contexto en el que existe una tendencia marcada entre los economistas a sólo leer aquello que publican otros economistas, dejando de lado lo desarrollado por otros científicos sociales, no obstante al parecer los demás científicos sociales (sociólogos, antropólogos, politólogos, historiadores, entre otros) sí leen lo que publican sus colegas de otras ciencias sociales, incluida la producción académica realizada por economistas (Fourcade et al., 2015).

\section{La noción de complejidad en la ciencia económica}

\subsection{Complejidad}

El concepto de complejidad incluye lo planteado en la Ley de la Entropía, llamada también Segunda Ley de la Termodinámica:

«(...) La complejidad no se define por la cantidad de variables o partes que intervienen en el funcionamiento de un sistema, sino como producto de una dinámica de la actuación unidireccional de la ley de entropía (...). La cantidad de definiciones que podemos encontrar en la literatura especializada sobre el tópico se debe a que la complejidad tiene algo de inasible: se refiere a un sinnúmero de fenómenos disímiles y en más de un sentido supera nuestras capacidades de comprensión. (...)» (Tyrtania, 2008: 43) ${ }^{9}$

El vínculo entre la idea de complejidad y caos (entropía) existe, pero la propia idea de caos no es absoluta, tiene la característica de ser relativa, ello es establecido por aquel que plantea cierto orden analítico, se entiende que en el tema de la investigación científica ello acontecerá. ${ }^{10}$ Lo presentado lo entendemos como parte de un acto de aducción, consideramos que estos conceptos propios de la Física, ayudan a realizar un planteamiento teórico en las Ciencias Sociales, enfocados en aspectos culturales. ${ }^{11}$

incluso los popperianos, desean, pero lo cierto es que la filosofía de la ciencia se ocupa, y se ha ocupado siempre, de cómo esas conjeturas iniciales se convierten en teorías científicas por medio de su inserción y articulación dentro de una estructura deductiva más o menos coherente y completa, y de cómo esas teorías son posteriormente contrastadas con las observaciones. En breve, no digamos que la ciencia se basa en la inducción: se basa en la aducción seguida de deducción.» (Blaug, 1997: 17) [La traducción es nuestra] Respecto a la aducción, puede verse un amplio desarrollo en Cisneros (2014c: 22-29).

9 Véase también: Rodríguez y Aguirre (2011), Cardozo (2011) y Cisneros (2014b).

10 Véase Georgescu-Roegen (1996: 198).

11 Véase Tyrtania(2008: 41). 


\section{Juan Manuel Eliud Cisneros García}

Para el programa científico de investigación dominante, llámesele PCI Ortodoxo, el mainstream, en el cual la Teoría Neoclásica es la principal, pareciera que la noción de caos, desorden, en cuanto al mundo social fuese de un grado más reducido, un nivel de complejidad menor, o en todo caso, que estuviese difundida la idea de que con los modelos existentes fuese suficiente para poder explicar cualquier hecho que apareciese en cualquier realidad, aplicacionismo. Nótese por ejemplo que en los modelos neoclásicos no existe mención, ni incorporación de la ley de la entropía, de hacerlo tendría que modificarse algunos de los supuestos principales, proposiciones de base $^{12}$, de la teoría señalada; no bastaría con añadir algunos supuestos auxiliares.

\subsection{Concepto de complejidad en las Ciencias Sociales}

Presentamos una aproximación de la definición de complejidad, se refiere a:

- El gran número y la heterogeneidad de los elementos que constituyen la realidad.

- Los factores que afectan los resultados de las relaciones entre los elementos son múltiples.

Las sociedades humanas constituyen realidades complejas y a la vez son sistemas complejos en los que interactúan individuos, en las que los individuos mismos son sistemas complejos. ${ }^{13}$

\section{Proceso analítico}

La noción básica de proceso ${ }^{14}$ implica la representación abstracta de una serie de

12 En la terminología planteada por Georgescu-Roegen, proposiciones alfa $(\alpha)$.

13 «(...) The simple fact that individuals in a human society are not identical, as compared to homogeneity of atoms in the physical world, suggests that the social world is more complex than the physical world. Human societies are complex systems of interacting individuals in which individuals themselves are complex systems.»(Figueroa, 2012: 26)

14 La palabra proceso tiene origen latino, del vocablo processus, de procedere, que viene de pro (para adelante) y cere (caer, caminar), ello tiene por significado: progreso, ir hacia adelante, avance, ir hacia un fin determinado. Según el Diccionario de la Real Academia Española (DRAE) la palabra proceso tiene las siguientes acepciones: «Acción de ir hacia adelante. Transcurso del tiempo. Conjunto de las fases sucesivas de un fenómeno natural o de una operación artificial. (...)». 


\section{Pensamiento Crítico Vol. 20. NoI}

actividades de duración determinada y repetidas, período tras período, orientadas a un fin. En las ciencias sociales, dichas actividades corresponden a un conjunto de hechos sociales observados, regularidades empíricas, dichos hechos son representados como un proceso.

La idea de proceso se establece por abstracción, previa aducción, si se considera a la realidad como un conjunto, una realidad total, el proceso abstracto sería un elemento de la realidad mencionada, por tanto sería un proceso parcial, lo cual implica asumir que la realidad puede dividirse en dos:

- Una representación del proceso parcial determinada por el interés de actualidad.

- El entorno de dicho proceso.

La separación está dada por un vacío aritmomórfico. De este modo, todo lo que sucede en la realidad en cualquier momento es parte bien del proceso en cuestión o de su entorno.

«Proceso es un concepto especialmente desconcertante, pues proceso es Cambio o no es nada en absoluto (...). El resultado final es que la ciencia debe tener una idea clara de cómo representar un proceso analíticamente. No hacerlo así antes de que empiece el juego puede convertirse en fuente de importantes errores. (...) En las ciencias sociales -especialmente en la economía, donde las argumentaciones teóricas sobre el papel tienen por regla general sólo un remoto contacto con los datos reales- "proceso» es un término del que se ha abusado en exceso: se emplea para indicar casi todo lo que uno desea. Sirva de testimonio de fórmulas matemáticas por las que se representa un elemento básico de la teoría económica como es el proceso de producción. Sirva igualmente de testimonio la falta de preocupación, prácticamente total, por lo que los símbolos de esas fórmulas representan en términos reales.» (Georgescu-Roegen, 1996: 275-276)

\subsection{Elementos de la imagen analítica del proceso}

La imagen analítica del proceso implica considerar dos elementos: el límite analítico y el suceso. 


\section{Juan Manuel Eliud Cisneros García}

\section{A. Límite analítico: Sin límite no hay proceso analítico}

El límite analítico está relacionado con la intencionalidad de trazo de límites del proceso. El estudio de la realidad ha compartimentado la realidad en campos de acuerdo a su intencionalidad, es decir en cada ciencia específica se ha trazado límites de procesos de acuerdo a donde conviene establecerlos en relación a su intencionalidad específica. ${ }^{15}$

El límite analítico consta de dos componentes: frontera del proceso y la duración del proceso. ${ }^{16}$

\section{a. Frontera del proceso}

La esencia del límite de proceso consiste de manera necesaria en establecer dos componentes analíticos distintos antes ya mencionados: el proceso y su entorno o frontera del proceso.

\section{b. Componente temporal del límite: duración del proceso}

En este punto resulta imprescindible la categoría de aritmomórfico, sin ella no se podría plantear la noción de instante de tiempo. ${ }^{17}$

Se deben especificar dos momentos: inicio y fin del proceso analítico que tenemos en mente, el componente temporal del proceso debe ser necesariamente un intervalo temporal finito:

Inicio del proceso: $t_{0} \quad$ sea: $t_{0}>-\infty$

Fin del proceso: $\quad t_{1} \quad$ sea: $t_{1}<+\infty$

15 Dicha intencionalidad puede estar basada en intereses económicos y políticos; al respecto existen serias críticas a lo hecho por los promotores de la teoría económica dominante, véase: Gonzáles (2004: 23-25)

16 «An analytical boundary, as conceived here, must consist of two components. Like a frontier, one component separates the process at any time from the rest of actually, although we must not think that this frontier is necessarily geographical in nature or rigidly determined. Witness the process of thought itself or than of an acorn growing into an oak. The second component is the duration of the process, determined by the time moments at which the process we have in mind begins and ends. Naturally, these moments must be at a finite distance; otherwise, we would not know all that has gone into the process or all that the process does.» (Georgescu-Roegen, 1970: 3)

17 Véase: Georgescu-Roegen (1996: 120). Respecto a la noción de proceso planteada por Whitehead y la teoría no estándar, véase Livadas (2013) 


\section{Pensamiento Crítico Vol. 20. NoI}

Se puede escoger la escala de tiempo, y expresar la duración del proceso que puede representarse por el intervalo temporal cerrado. $\left[t_{0}, t_{1}\right]$

Se considera que el proceso analítico no tiene existencia para $t<t_{0}$ y $t>t_{1}$, para fines de especificación del proceso, no se considera lo acontecido antes de $t_{0} y$ después de $t_{1}$, es decir se hace abstracción de lo que pueda haber acontecido en la realidad antes de $t_{0}$ y lo que sucederá después de $t_{1}$

No deberían ser considerados como proceso analítico:

Si $t_{0}=-\infty$, no se sabría todo lo que ha entrado al proceso.

Si $t_{1}=+\infty$, no se sabría todo lo que hace el proceso.

Si $t_{0}=t_{1}$, sería un proceso sin duración. ${ }^{18}$

En base a la idea de duración finita, tampoco puede existir un proceso de duración: $<-\infty,+\infty>$

\section{B. Suceso: imposibilidad de explicar lo que ocurre dentro del proceso}

Un proceso implica algún suceso, por tanto éste debe ser representado analíticamente.

La identificación del proceso en base a sus límites, implica la idea de no describir que es lo que acontece dentro del proceso, dentro de los límites del proceso abstracto, si se pretendiera ello, se presentarían dos posibles situaciones:

- Se tendría que trazar otro límite transversal al proceso y dividir éste en dos procesos: el proceso inicial y su interior, los cuales tendrían que ser estudiados por separado; esos dos procesos no podrían haber sido parte de la imagen analítica inicial (antes de haberse trazado el nuevo límite), es decir ya no

18 «(...) Georgescu-Roegen argued that the analytical boundary consists of two components, one that separates the process from the rest of reality while the other specifies the duration of the process, because a timeless process is obviously a contradictio in adjecto. It comes as no surprise that he was opposed to the concept of instantaneous production as encountered, for example, in Walras.»(Kurz y Salvadori, 2003: 490) 


\section{Juan Manuel Eliud Cisneros García}

se tendría únicamente el proceso que inicialmente se pretendía explicar, se tendría la existencia de uno adicional.

- Si se enfocase el interés en realizar una descripción de todo lo que sucede en el interior del proceso (dentro de él), se llegaría a una regresión infinita o regresión al infinito (regressus ad infinitum). En un proceso existe un mecanismo subyacente inobservable, por el cual los elementos exógenos afectan a los elementos endógenos; lo que sucede al interior del proceso, es decir al interior del mecanismo, no puede ser observado; si fuera observado, éste podría ser considerado como un proceso en sí mismo con elementos endógenos y exógenos, y un interior inobservable y así podríamos retroceder en una regresión continua al infinito. ${ }^{19}$

\subsection{Las coordenadas analíticas de un proceso}

\section{A. Tipos de elementos que cruzan los límites del proceso $(C)$}

La descripción de lo que acontece en conexión con un proceso se reduce a registrar lo que cruza los límites del mismo. Sea: $\mathrm{C}$ el conjunto de elementos que pueden cruzar los límites de un proceso determinado $\left(\mathrm{C}_{1}, \mathrm{C}_{2}, \ldots, \mathrm{C}_{\mathrm{n}}\right)$

En un proceso existen dos tipos de elementos que cruzan sus límites, clasificación de $C$ :

\section{a. Inputs (entradas) o elementos exógenos $(F)$}

Elementos que cruzan los límites desde el contorno hacia el proceso, los que ingresan al proceso.

\section{b. Outputs (salidas) o elementos endógenos (G)}

Elementos que cruzan los límites del proceso hacia fuera, los que salen, son resultado del proceso. Los elementos endógenos del proceso constituyen el objeto de estudio de la ciencia respectiva.

$$
C=\{F, G\}
$$

19 «En última instancia, debe existir algo escondido detrás de las cosas que observamos. La ciencia busca entonces descubrir estos elementos subyacentes». (Figueroa, 2003: 32) 


\section{Pensamiento Crítico Vol. 20. NoI}

La descripción analítica es completa si para cada $C_{i}$ se determinan dos funciones no decrecientes:

$\mathrm{F}_{\mathrm{i}}(t)$ Muestra la entrada acumulativa hasta el momento $t$.

$\mathrm{G}_{\mathrm{i}}(t)$ Muestra la salida acumulativa hasta el momento $t$.

$C_{i}=\left\{F_{i}(t), G_{i}(t)\right\}$

Ambas funciones deben estar definidas para toda la duración del proceso es decir, el intervalo temporal cerrado $\left[t_{0}, t_{1}\right]$, antes ya mencionado.

De manera más específica:

$C_{i}=\left\{F_{i}\left(t_{0}\right), G_{i}\left(t_{1}\right)\right\}$

\section{B. Flecha del tiempo: Irreversibilidad de los procesos}

Vinculada con la duración del proceso, la presencia de los elementos $C$ se encuentra relacionada con la noción de flecha del tiempo ${ }^{20}$, ésta indica la dirección irreversible de los procesos.

Si vinculamos este punto en el proceso productivo, encontramos la idea de irreversibilidad de la producción. Los procesos no se pueden operar hacia atrás, no es posible cambiar la ubicación de los inputs y de los outputs en el proceso de producción, ello es central en el modelo planteado por Georgescu-Roegen en lo referido a la producción, el Modelo de Fondo-Flujo ${ }^{21}$, en el cual las leyes de la termodinámica están presentes.

Lo mencionado puede representarse según lo planteado en el Gráfico 1.

20 «La flecha del tiempo es un término acuñado por Arthur S. Eddington para indicar la dirección irreversible de los procesos de transformación de la energía debido al aumento de entropía exigido por la segunda ley. (...)». (Tyrtania, 2008: 42-43)

21 No es la intención del presente escrito plantear de manera detallada el modelo en mención, para una revisión básica del tema puede revisarse Zamberlan (2010: 103). Para una revisión con mayor detalle, véase: Georgescu-Roegen (1966, 1979 y 1996). Un modelo desarrollado basado en la propuesta de Georgescu-Roegen respecto al proceso de producción en el análisis económico puede revisarse en: Figueroa (1996: Capítulo 2) 


\section{Juan Manuel Eliud Cisneros García}

Gráfico 1: Representación diagramática de un proceso.

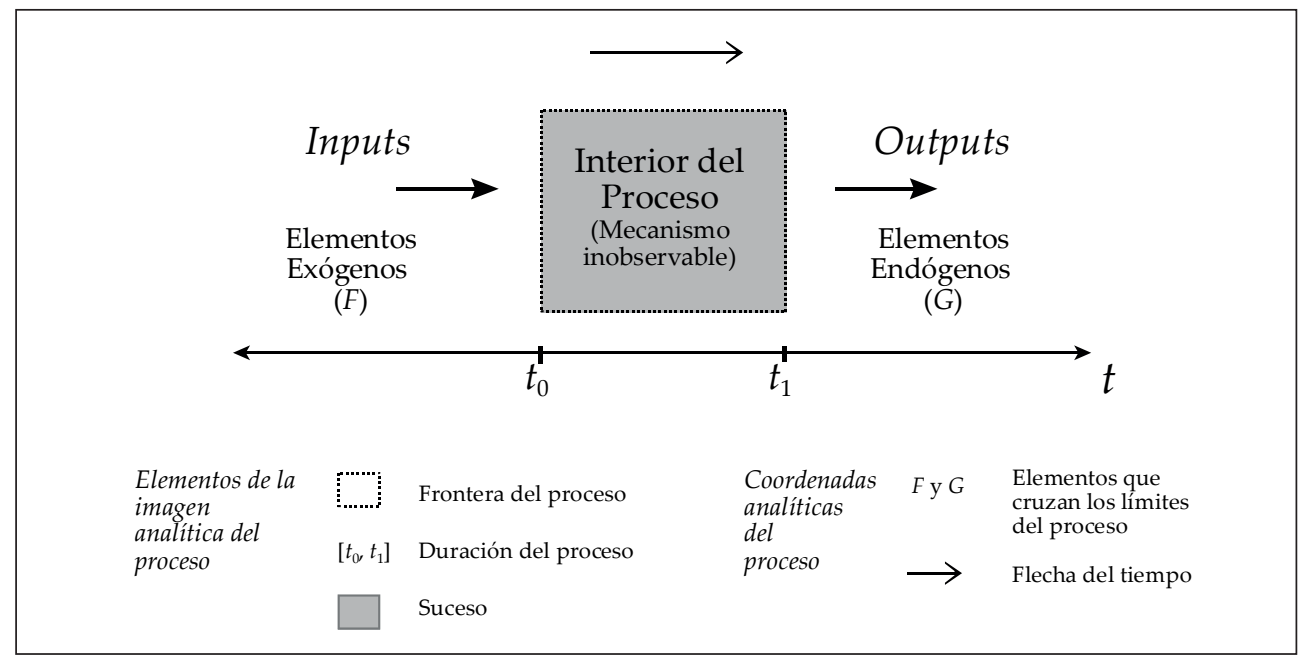

Fuente: Elaboración propia.

\subsection{Un elemento no siempre es una variable}

Los elementos $C$ deben poder ser expresados en unidades de medida, si ello no ocurriese no serán incluidos como parte de la explicación, pues no pueden ser expresados como una regularidad empírica, no habría proceso.

Los elementos exógenos $(F)$ y endógenos $(G)$ deben ser medibles, es decir deben ser variables. En el proceso abstracto, un aspecto central es el de establecer diferencia entre:

- Los elementos observables (medibles): serán llamados variables.

- Los elementos inobservables ${ }^{22}$ (no medibles).

22 Un ejemplo de elemento no medible es el siguiente enunciado que plantearía un chamán a uno de sus clientes: "Si tienes fe, mi medicina te sanará". «Si el cliente no se sana, el chamán le dirá que no tuvo fe. Como la fe no es observable, el cliente no tendrá manera de probarle a este chamán que su medicina es inútil. Esta proposición es, en realidad una tautología, pues siempre será verdadera, no hay manera de refutarla.» (Figueroa, 2008: 25) 


\section{Pensamiento Crítico Vol. 20. NoI}

\section{Noción de proceso e importancia en la ciencia económica}

La noción de complejidad que caracteriza los hechos sociales no permite plantear un adecuado entendimiento de éstos, por ello es necesario que dichos hechos puedan ser representados como un proceso.

\subsection{Regularidades empíricas: relaciones entre elementos exógenos y endógenos}

La ocurrencia de regularidades respecto a un hecho (o conjunto de hechos de la realidad), un fenómeno real, es condición necesaria para que éste sea sujeto de investigación científica. Dada que los hechos de un proceso se repiten período tras período, las relaciones entre los elementos endógenos y los exógenos pueden ser observadas empíricamente de manera continua, ello posibilita la ocurrencia de relaciones sistemáticas o regularidades. Lo fundamental es la existencia de datos históricos. Si fuese el caso de un mundo caótico, en el que las regularidades estuviesen ausentes de un proceso, ello presentaría dificultades para ser objeto de investigación científica.

\section{A. Se refieren al agregado del comportamiento de los individuos}

Las regularidades empíricas únicamente están referidas al agregado del comportamiento de los individuos, respecto al comportamiento de cada individuo no es esperable la aparición de regularidades. Las desviaciones individuales tienden a compensarse en el agregado.

\section{B. Son leyes estadísticas}

Las regularidades empíricas son leyes estadísticas, son válidas en la mayoría de casos, pero no en todos. Las regularidades en la Ciencia Económica no son leyes determinísticas, no son siempre válidas. Si se cuenta con una base de datos respecto a algunas variables se puede observar el comportamiento de éstas, ello no significa que se esté realizando una explicación sobre las relaciones existentes entre ellas. Por ejemplo si se tratase de asuntos distributivos, se puede iniciar realizando el cálculo de algunas medidas de resumen: media aritmética (promedio), mediana, cuantiles y proporciones; se podrá apreciar la relación entre la media y mediana, si bien la media es una medida más popular que la mediana, esta última tiene una 


\section{Juan Manuel Eliud Cisneros García}

propiedad interesante: es considerablemente más robusta a la presencia de valores atípicos (outliers). Entre otros aspectos. ${ }^{23}$

\subsection{Leyes económicas - Hechos estilizados - Hechos observados}

Las regularidades empíricas suelen también ser denominadas leyes económicas. En algunos casos las regularidades empíricas suelen ser presentadas bajo el nombre hechos estilizados o incluso hechos observados. En gran parte de los casos incluso se asigna el nombre del supuesto economista o pensador que se percató de tal ley estadística, es decir aparece el epónimo o la eponimia. ${ }^{24}$

\subsection{Hechos investigables y no investigables en la ciencia económica}

Existen aspectos de la realidad que no son investigables y otros que sí lo son, no todo hecho social puede ser explicado bajo las reglas del conocimiento científico, sólo son investigables aquellos que muestren relaciones sistemáticas, regularidades empíricas, que pueden ser representados como un proceso abstracto.

No es investigable:

- Algo que no existe no es investigable, no se puede investigar un hecho que no ha ocurrido, que no ha acontecido en realidad alguna.

- No se pueden investigar hechos de sociedades no reales, realidades netamente imaginarias.

- Aquello que se espera suceda en el futuro, pues aún no ha acontecido. Tal vez resulten importantes para algunos hoy los hechos futuros, pero precisamente hoy no son investigables, pues no existen (los serían si llegasen a ocurrir y se pudieran expresar como un proceso).

- Un hecho de única aparición (circunstancial o incluso anecdótico).

23 Para tener una idea más amplia de lo mencionado, puede verse: Gasparini et al. (2012: Capítulo 2).

24 «(...) Según R.K. Merton, la "eponimia" es "la práctica de adherir el nombre del científico a todo o parte de lo que ha encontrado" (...)» (Blaug, 2001: 588). 


\section{Pensamiento Crítico Vol. 20. NoI}

- Tampoco son investigables los casos en los cuales la duración del proceso, $t$, es ilimitada ya sea al inicio o al final: $t_{0}=-\infty$, ni $t_{1}=+\infty$; ni cuando la duración del proceso es igual a cero: $t_{0}=t_{1}$.

El motivo por el cual no son investigables es que para ninguno de los casos mencionados existen datos históricos sobre los que se pueda explicar el hecho, es decir no puede observarse regularidad empírica alguna, por tanto no existe proceso (como ya se indicó: una serie de actividades, de una determinada duración, orientadas a un fin y repetidas período tras período), no se puede plantear un proceso analítico.

No todo lo que se considera importante o útil es investigable en la Ciencia Económica. Sólo es investigable aquello que ya ocurrió, lo que es investigable son los hechos de los que existen datos; algo similar ocurre en las otras ciencias fácticas, incluidas las ciencias naturales.

\section{Sin proceso analítico, no existe teoría; sin teoría no hay explicación}

La explicación del resultado de un proceso analítico se realiza planteando una teoría. Una ciencia social está interesada en explicar el resultado de un proceso abstracto, el cual corresponde a ciertas regularidades empíricas. Los elementos endógenos de dicho proceso constituyen el objeto de estudio de la ciencia social respectiva, en tanto que los elementos exógenos y los mecanismos de interacción entre estos y aquellos son los que permiten explicar los fenómenos, y son establecidos, ambos, mediante supuestos.

\subsection{Investigación teórica}

Entendemos que en el tema de investigación en la Ciencia Económica, en la actualidad, existen dos tendencias: investigación teórica e investigación empírica. Lo expuesto hasta el momento corresponde a la primera, la investigación empírica no es tema del presente escrito ${ }^{25}$. La investigación teórica en la Ciencia Económica

25 De manera muy concreta, entendemos por investigación empírica (empirical research) el planteamiento de un conjunto de ecuaciones, que puede ser sólo una (basadas en algunos casos en una teoría económica, a veces no es tal la situación), a partir de ello se hace uso de un modelo econométrico (casi siempre paramétrico) y haciendo uso de una pertinente base de datos y un adecuado software informático (Stata, EViews, R, otros) se busca "explicar" (lo correcto es indicar que se busca predecir) aspectos de una realidad. 


\section{Juan Manuel Eliud Cisneros García}

busca generar conocimiento científico sobre el funcionamiento del mundo social, es decir explicar aquellos hechos ya acontecidos en una realidad, los cuales se han presentado de manera repetida, como un proceso, siendo por ello posible apreciar algunas regularidades. El porqué y para qué se requiere realizar investigación desde la perspectiva de la ciencia es que precisamente la investigación es un componente importante para llegar al conocimiento científico sobre el funcionamiento del mundo social, los conceptos mencionados: abstracción, aducción, aritmomórfico y proceso analítico resultan importantes para lograr ello.

Gráfico 2: Investigación teórica - idea básica.

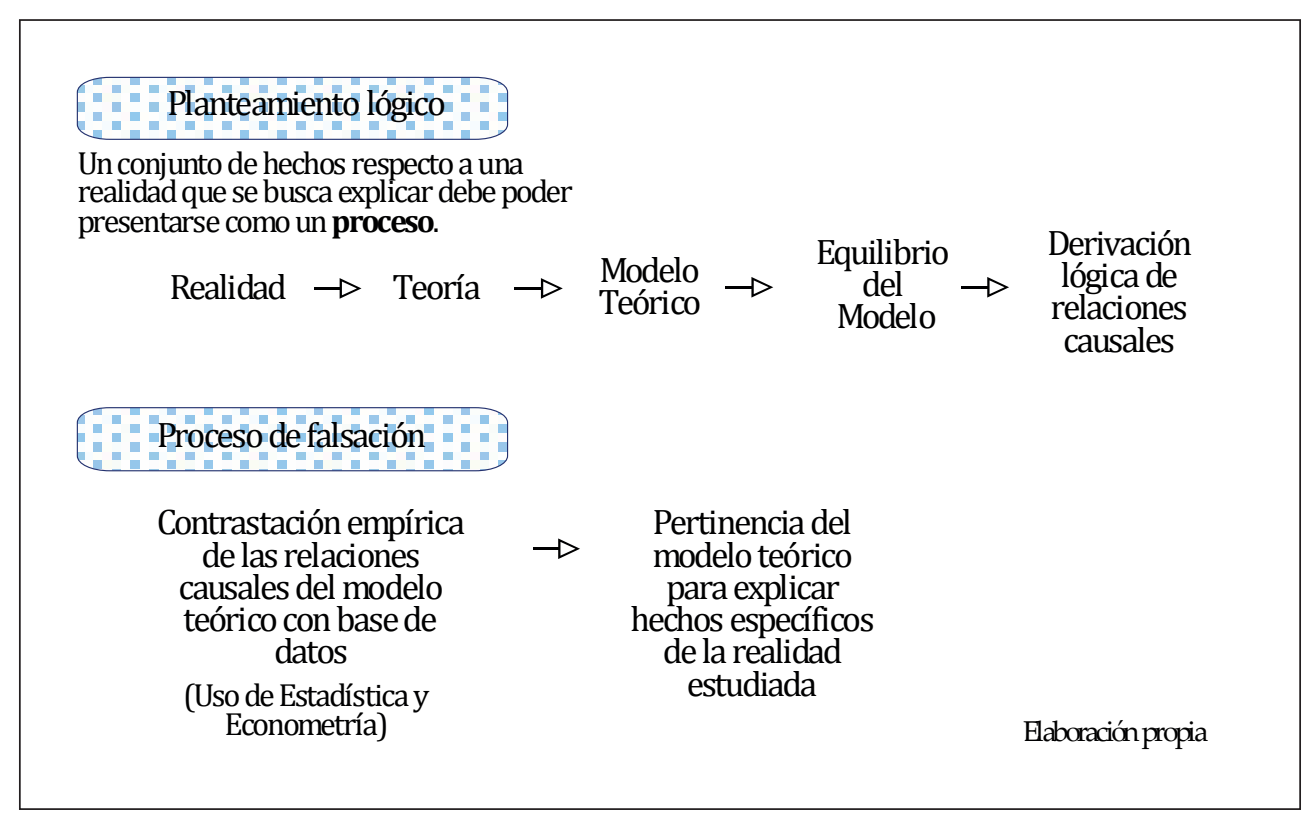

El Gráfico 2 permitirá explicar algunas ideas sobre los pasos que se consideran para el desarrollo de la investigación teórica. Se aprecia la existencia de una base de datos al inicio y al final. Al inicio, la base de datos permite establecer las regularidades empíricas, si se presentase sólo algunos cuadros y gráficos no se realiza explicación alguna, tan sólo se podría narrar los cambios en las variables que aparecen en ellos ${ }^{26}$. En la parte final

26 Tal como ocurre con el periodismo económico. Diversos artículos que indican que una variable aumentó o disminuyó, pero sin explicar los motivos. 


\section{Pensamiento Crítico Vol. 20. NoI}

de la investigación se requiere una base de datos pues se debe realizar la contrastación empírica de las hipótesis planteadas en los modelos teóricos ${ }^{27}$, los modelos permiten hacer operativa dicha teoría ${ }^{28}$, es importante recordar que en la Ciencia Económica la existen un conjunto de teorías, no sólo una, todas han sido establecidas de manera arbitraria por alguna persona o conjunto de individuos ${ }^{29}$, por tanto es fundamental la contrastación empírica (proceso de falsación) para lo cual se requiere el conocimiento de Estadística y Econometría, en ambos casos lo no paramétrica es lo más pertinente.

\section{Conclusiones}

Lo indicado respecto a la investigación teórica debería llevar a realizar una evaluación de la manera como se desarrollan las investigaciones económicas en la actualidad en las Facultades de Ciencias Económicas. Así como el desarrollo de las actividades lectivas en asignaturas que se relacionan con algunas teorías, en el campo de economía ortodoxa, cursos tales como Microeconomía, Macroeconomía y afines, y en el campo de la economía no ortodoxa: Economía Política, entre otros. No se puede dejar de lado aquellas asignaturas vinculadas con aspectos empresariales que suelen ser también parte de los planes de estudios de diversas Facultades de Ciencias Económicas, cursos tales como Finanzas, Investigación de Mercados, Marketing, Planeamiento Estratégico, entre otros, debería primero evaluarse si realmente se desarrollan actividades de investigación a partir de ellas. ${ }^{30}$

27 Los cuales se formulan a partir de una teoría específica, se incorporan supuestos a los que ya existentes en una teoría económica. Al respecto se recomienda revisar: Figueroa (2003: Capítulo 1) y Figueroa (1996: Capítulo 1 y Apéndice)

28 Una teoría está conformada por un conjunto de modelos.

29 Véase Cisneros (2014a)

30 Más allá de lo que indiquen los títulos de los documentos, e incluso los propios docentes. La gran parte de dichas "investigaciones" no pueden ser incluidas como parte del conjunto de la investigación teórica, ni tampoco de la investigación empírica. Hacemos la acotación pues entendemos aún existe confusión o desconocimiento en el tema, recordamos el caso de un Decano de una Facultad de Ciencias Económicas de una Universidad importante de Perú intentando argumentar que lo que se hace en dichas disciplinas es "otro tipo de investigación", lo importante era que no indicaba a cuál se refería, ni podía argumentar ello; lo indicado es una muestra de que los aspectos epistemológicos sí resultan fundamentales, y útiles, en la Ciencia Económica, aunque en la actualidad se haya relegado su importancia. 


\section{Juan Manuel Eliud Cisneros García}

\section{Referencias bibliográficas}

Black, M. (1970). Margins of precision. Essays in logic and language. Ithaca: Cornell University Press.

Blaug, M. (2001). Teoría económica en retrospección. 5a ed. México, D.F.: Fondo de Cultura Económica.

Blaug, M. (1997). The methodology of economics or how economists explain. $2^{\mathrm{a}}$ ed. Cambridge: Cambridge UniversityPress.

Cardozo, M. (2011). Las ciencias sociales y el problema de la complejidad. Argumentos, estudios críticos de la sociedad, 24(67), 15-38.

Cisneros, J. (2014a). Apuntes sobre la metodología alfa beta: las proposiciones alfa. Apunte de Clase No 3. Lima: Escuela Académico Profesional de Economía, Facultad de Ciencias Económicas, Universidad Nacional Mayor de San Marcos.

Cisneros, J. (2014b). La noción de proceso y su importancia en la investigación en la ciencia económica. Documentos de Trabajo Omega Beta Gamma № 05. Lima: Facultad de Ciencias Económicas, Universidad Nacional Mayor de San Marcos.

Cisneros, J. (2014c). Las proposiciones alfa: notas sobre la propuesta epistemológica planteada por Georgescu-Roegen. Documentos de Trabajo Omega Beta Gamma No 08. Lima: Facultad de Ciencias Económicas, Universidad Nacional Mayor de San Marcos.

Dulbecco, P. y Garrouste, P. (2004). Théorie de la dynamique économique une réévaluation de la tentative de Nicholas Georgescu-Roegen. Recherches Économiques de Louvain - Louvain Economie Review, 70(1), 5-29.

Encinas, J. (1992). Filosofía, teoría y metodología en la ciencia económica. Moneda, 4(44), 24-34.

Figueroa, A. (1996). Teorías económicas del capitalismo. $2^{a}$ ed. Lima: Fondo Editorial de la Pontificia Universidad Católica del Perú - Fondo de Cultura Económica. 


\section{Pensamiento Crítico Vol. 20. NoI}

Figueroa, A. (2003). La sociedad sigma: una teoría del desarrollo económico. Lima: Fondo Editorial de la Pontificia Universidad Católica del Perú - Fondo de Cultura Económica.

Figueroa, A. (2008). Nuestro mundo social. Introducción a la ciencia económica. Lima: Fondo Editorial de la Pontificia Universidad Católica del Perú.

Figueroa, A. (2012). A unified theory of capitalist development: growth, inequality and the environment. Edición revisada y ampliada. Buenos Aires: Cengage Learning.

Fontini, F. (2005). Probability and uncertainty: the legacy of Georgescu-Roegen. Marco

Fanno Working Paper $N^{\circ}$ 8. Padova: Dipartimento di Scienze Economiche Marco Fanno, Università degli Studi di Padova.

Fourcade, M.; Ollion, E. y Algan, Y. (2015). The superiority of economists. Journal of Economic Perspectives, 29(1): 89-114.

Gasparini, L.; Cicowiez, M. y Sosa Escudero, W. (2012). Pobreza y desigualdad en América Latina: conceptos, herramientas y aplicaciones. Buenos Aires: Temas Grupo Editorial - Centro de Estudios Distributivos, Laborales y Sociales (CEDLAS), Universidad Nacional de La Plata.

Georgescu-Roegen, N. (1966). Analytical economics, issues and problems. Cambridge: Harvard University Press.

Georgescu-Roegen, N. (1979). Energy analysis and economic evaluation. Southern Economic Journal, 45(4), 1023-1058.

Georgescu-Roegen, N. (1986). Métodos em Ciência Econômica. Economia-Ensaios, Uberlândia, 20(1): 7-16.

Georgescu-Roegen, N. (1996). La ley de la entropía y el proceso económico. Madrid: Fundación Argentaria - Visor Distribuciones. 


\section{Juan Manuel Eliud Cisneros García}

Gonzáles, P. (2004). Las nuevas ciencias y las humanidades. De la academia a la política. Madrid: Editorial Complutense - Anthropos Editorial - Instituto de Investigaciones Sociales (UNAM)

Kurz, H. y Salvadori, N. (2003). Fund-flow versus flow-flow in production theory: reflections on Georgescu-Roegen's contribution. Journal of Economic Behavior \& Organization, 51, 487-505.

Livadas, S. (2013). The notion of process in nonstandard theory and in whiteheadian metaphysics. Manuscrito, Rev. Int. Fil., Campinas, 36(1), 103-137.

Popper, K. (2002). The logic of scientific discovery. Londres: RoutledgeClassics.

Rodríguez, L. y Aguirre, J. (2011). Teorías de la complejidad y ciencias sociales. Nuevas estrategias epistemológicas y metodológicas. Nómadas. Revista Crítica de Ciencias Sociales y Jurídicas, 30(2), 1-20.

Tyrtania, L. (2008). La indeterminación entrópica. Notas sobre disipación de energía, evolución y complejidad. Desacatos, 28, 41-68.

Varian, H. (1997). How to build an economic model in your spare time. En: Szenberg, M. (ed.). Passion and craft: economists at work. Michigan: University of Michigan Press.

Zamberlan, S. (2010). From utility to the enjoyment of life: the bioeconomics of Nicholas Georgescu-Roegen. The influence on the italian authors. En: Galindo, M. y Nardi, C. (eds.). Issues in economic thought. Nueva York: Nova Science Publishers, Inc., 99-116. 